\title{
Dual bronchodilation and exacerbations of COPD
}

\author{
Mario Cazzola, Josuel Ora, Ermanno Puxeddu \\ Department of Systems Medicine, University of Rome Tor Vergata, Rome, Italy \\ Correspondence to: Prof. Mario Cazzola. Department of Systems Medicine, University of Rome Tor Vergata, Rome, Italy. \\ Email: mario.cazzola@uniroma2.it.
}

Submitted Jul 26, 2016. Accepted for publication Aug 02, 2016.

doi: $10.21037 /$ jtd.2016.08.92

View this article at: http://dx.doi.org/10.21037/jtd.2016.08.92

In the 2016 updated version of Global Initiative for Chronic Obstructive Lung Disease (GOLD) report, it is stated that combinations of a long-acting $\beta_{2}$-agonist (LABA) and a long-acting antimuscarinic agent (LAMA) significantly increase lung function but the impact on patient reported outcomes is limited (1). Furthermore, it also affirms that there is still too little evidence to determine if dual bronchodilation is more effective than a LAMA alone for preventing acute exacerbations of COPD (AECOPDs) (1). This sentence is inexplicable because when the 2016 version of the GOLD report was written, there was already some published evidence showing that dual bronchodilation with a LABA and a LAMA has greater efficacy in the reduction of AECOPD rates than does LAMA monotherapy (2).

Anyway, both of these opinions have been overtaken, or at least questioned, by the recent acquisition of new important information delivered to literature.

With regard to the first statement, we must highlight that we have carried out a systematic review with metaanalysis that incorporated the data from trials lasting at least 3 months to evaluate the effectiveness of LAMA/LABA fixed-dose combinations (FDCs) for COPD treatment. Our results showed that all LAMA/LABA FDCs were always more effective than the LAMA or LABA alone in terms of improvement in trough $\mathrm{FEV}_{1}$, transitional dyspnea index (TDI) and St. George's Respiratory Questionnaire (SGRQ) scores (3). Consequently, they suggested that it is appropriate to always consider that the dual bronchodilation is always better than a LAMA or a LABA alone, regardless of the drugs used.

This solid evidence, combined with the documentation produced by our recent preclinical studies demonstrating that combining a LABA with a LAMA provides synergistic benefit on airway smooth muscle relaxation (4), which may have major implications for the use of LABA/LAMA FDCs in the treatment COPD (5), supports the possibility of an early intervention with low doses of LABA/LAMA FDC to optimize bronchodilation and reduce the risk of adverse events that characterize both LABAs and LAMAs, especially when administered at the full doses currently approved for the treatment of COPD (6).

With regard to the second statement, the very recent large FLAME study (7), a 52-week, randomized, doubleblind, double-dummy, noninferiority trial that enrolled 3,362 patients who had COPD with a history of at least one exacerbation during the previous year and were randomly assigned to receive, by inhalation, either indacaterol $(110 \mu \mathrm{g})$ plus glycopyrronium $(50 \mu \mathrm{g})$ once daily or salmeterol $(50 \mu \mathrm{g})$ plus fluticasone $(500 \mu \mathrm{g})$ twice daily, showed that dual bronchodilation was more effective than LABA/ICS FDC in preventing AECOPDs in these patients. Besides, the change from baseline in trough $\mathrm{FEV}_{1}$ was significantly greater in the indacaterol/glycopyrronium group than in the salmeterol/fluticasone group.

We must emphasise that findings supporting the use of the indacaterol/glycopyrronium FDC as an alternative treatment, over salmeterol/fluticasone FDC, in the management of moderate-to-severe COPD patients without AECOPDs in the previous year (8) and with a history of $\leq 1$ AECOPD in the previous year (9) had already been delivered to the literature. Furthermore, clinical evidence demonstrated that indacaterol/glycopyrronium FDC delayed the time to first AECOPD when compared with salmeterol/fluticasone FDC $(9,10)$. In particular, in patients with a history of $\leq 1$ AECOPD in the previous year (9), the hazard ratio for the time to first moderate or severe AECOPD was significantly delayed although almost half the cases were classified as severe (GOLD D) and had 
a lower mean postbronchodilator $\mathrm{FEV}_{1}$ at baseline when compared to patients without AECOPDs in the previous year (8).

In any case, the greater value of the FLAME trial compared to previous studies is that it was primarily focused on AECOPDs and correctly lasted 52-week, which represents the minimum period of duration of a trial focused on AECOPDs (11).

If dual bronchodilation is effective in preventing AECOPDs, we need to understand the mechanism(s) that explain(s) this action. Wedzicha and colleagues believe that the most important mechanism involved in the effect of bronchodilators on AECOPDs probably involves reduction of hyperinflation and a re-setting of lung function dynamics (12). Both indacaterol and glycopyrronium are able to induce potent, significant and long-lasting relaxation of both medium and small human isolated bronchi pre-contracted with acetylcholine (13). However, the co-administration of glycopyrronium and indacaterol produces a synergistic inhibition of the entire airway smooth muscle tone via modulating the cAMP-dependent pathway. The greater effectiveness of the indacaterol/glycopyrronium combination on small airways, compared with the partial effect of glycopyrronium or indacaterol alone, might be of particular clinical relevance for improving air-trapping related to the obstruction of bronchioles. In fact, as airway patency over time increases with longer duration of a more potent bronchodilator action, emptying of peripheral airways with trapped air is facilitated, thus reducing hyperinflation and improving breathing mechanics ("pharmacological lung volume reduction") (14), and consequently reducing the risk of AECOPDs.

Although this is an elegant explanation, we believe that the reduced release of acetylcholine from the epithelium but not from bronchi caused by the co-administration of indacaterol and glycopyrronium (13) is even more important to explain the ability of dual bronchodilation in preventing AECOPD. Epithelial cells, which may express choline acetyltransferase (ChAT), are a source of non-neuronal acetylcholine in the airways in response to inflammatory stimuli (15). Non-neuronal acetylcholine has important inflammatory properties (16). The multitude of cells in the airways involved in AECOPD, including bronchial epithelial cells, neutrophils, lymphocytes, macrophages and fibroblasts, have muscarinic receptors (17).

However, regardless of the mechanism, the evidence that dual bronchodilation can prevent or at least delay the onset of AECOPD raises the fundamental questions whether it makes sense to switch all patients from a LABA/ ICS regimen to a LABA/LAMA regimen on the basis of the improvement in lung function and the lower exacerbation rates (18) or there is a subgroup of patients with COPD who may benefit the most from this therapy.

In the past, considering the published evidence, we suggested that those patients that have suffered from mild AECOPDs should be treated regularly with a dual bronchodilation therapy, whereas those that have been treated with oral corticosteroids because of their AECOPD should be treated with LABA/ICS, and all patients hospitalised because an AECOPD should be discharged with the prescription of long-term triple therapy (6).

Unfortunately, the FLAME study does not allow to clarify the real value of preventing AECOPDs by treating patients according to the reported severity of exacerbations. A subgroup analysis of the rate of all exacerbations in the FLAME study showed that indacaterol/glycopyrronium was better than salmeterol/fluticasone in patients included in GOLD group B and D (i.e., high-risk patients) regardless of use of ICS or LABA/ICS at the screening, but salmeterol/ fluticasone was slightly better in patients with very severe airflow limitation (GOLD 4) (7). Furthermore, there was no difference between the two treatments in preventing mild exacerbations but indacaterol/glycopyrronium was more effective that salmeterol/fluticasone in preventing moderateto-severe exacerbations (7). These findings suggest that we should always prefer dual bronchodilation when we are focused on the reported severity of exacerbations for preventing further AECOPDs. Nonetheless, we strongly believe that further studies are needed to consolidate this evidence and clarify which characteristics of the patient with COPD should help us in selecting the most appropriate therapy.

In particular, we would like to know if dual bronchodilation is effective in preventing AECOPDs regardless of their nature. In fact, four distinct biological exacerbation clusters were identified: bacterial-, viral-, and eosinophil-predominant, and a fourth associated with limited changes in the inflammatory profile termed "pauciinflammatory" (19). In any case, in the FLAME study, the baseline blood eosinophil count [blood eosinophil count $\geq 2 \%$ is a promising biomarker of response to ICSs in patients with COPD (20)], did not appear to predict what would be the most effective treatment regimen (7).

Alternatively, it would be possible to hypothesize the use of dual bronchodilation based on COPD 
phenotype (21). The prescription of dual bronchodilator therapy should always be preferred in the emphysemahyperinflation phenotype (6), in which the already mentioned "pharmacological lung volume reduction" (14) is extremely important. In frequent exacerbators, mainly those with a history of two or more exacerbations during the previous year that frequently present with chronic bronchitis, defined as the presence of productive cough or expectoration for $>3$ months per year and $>2$ consecutive years and are the only subjects with an indication for antiinflammatory treatment in COPD, the treatment is based on a LABA/ICS combination (22). However, the results of the FLAME study, which was not really focused on different COPD phenotypes, suggest that this recommendation is not completely correct because in frequent exacerbators, indacaterol/glycopyrronium seemed to be more effective than salmeterol/fluticasone (7).

The documentation that dual bronchodilation may prevent AECOPDs even in the absence of an ICS in frequent exacerbators raises another fundamental question, which patients with COPD can benefit from therapy with ICSs. Moreover, it is essential to establish whether LABA/LAMA combination therapy is preferred over triple therapy (LAMA/LABA/ICS), and whether addition of an ICS to the LABA/LAMA combination provides additional clinical value because data are still too scarce and studies too short to generate a strong recommendation despite the publication of the WISDOM study (23).

\section{Acknowledgements}

M Cazzola is a consultant and a member of the Speaker Bureau for Novartis.

\section{Footnote}

Provenance: This is an invited Editorial commissioned by the Section Editor Yan Xu (Department of Respiratory Medicine, Peking Union Medical College Hospital, Peking Union Medical College, Chinese Academy of Medical Sciences, Beijing, China).

Conflicts of Interest: The authors have no conflicts of interest to declare.

Comment on: Wedzicha JA, Banerji D, Chapman KR, et al. Indacaterol-Glycopyrronium versus Salmeterol-Fluticasone for COPD. N Engl J Med 2016;374:2222-34.

\section{References}

1. Global Strategy for the Diagnosis, Management and Prevention of COPD. Global Initiative for Chronic Obstructive Lung Disease. Available online: http:// goldcopd.org/

2. Wedzicha JA, Decramer M, Ficker JH, et al. Analysis of chronic obstructive pulmonary disease exacerbations with the dual bronchodilator QVA149 compared with glycopyrronium and tiotropium (SPARK): a randomised, double-blind, parallel-group study. Lancet Respir Med 2013;1:199-209.

3. Calzetta L, Rogliani P, Matera MG, et al. A Systematic Review With Meta-Analysis of Dual Bronchodilation With LAMA/LABA for the Treatment of Stable COPD. Chest 2016;149:1181-96.

4. Calzetta L, Matera MG, Cazzola M. Pharmacological interaction between LABAs and LAMAs in the airways: optimizing synergy. Eur J Pharmacol 2015;761:168-73.

5. Cazzola M, Calzetta L, Segreti A, et al. Translational Study Searching for Synergy between Glycopyrronium and Indacaterol. COPD 2015;12:175-81.

6. Cazzola M, Rogliani P, Matera MG. Escalation and Deescalation of Therapy in COPD: Myths, Realities and Perspectives. Drugs 2015;75:1575-85.

7. Wedzicha JA, Banerji D, Chapman KR, et al. IndacaterolGlycopyrronium versus Salmeterol-Fluticasone for COPD. N Engl J Med 2016;374:2222-34.

8. Vogelmeier CF, Bateman ED, Pallante J, et al. Efficacy and safety of once-daily QVA149 compared with twice-daily salmeterol-fluticasone in patients with chronic obstructive pulmonary disease (ILLUMINATE): a randomised, double-blind, parallel group study. Lancet Respir Med 2013;1:51-60.

9. Zhong N, Wang C, Zhou X, et al. LANTERN: a randomized study of QVA149 versus salmeterol/fluticasone combination in patients with COPD. Int J Chron Obstruct Pulmon Dis 2015;10:1015-26.

10. Banerji D, Fedele MJ, Chen H, et al. Dual bronchodilation with QVA149 reduces COPD exacerbations: results from the IGNITE program (OS36: COPD 3). Chest 2014;145:403A.

11. Cazzola M, MacNee W, Martinez FJ, et al. Outcomes for COPD pharmacological trials: from lung function to biomarkers. Eur Respir J 2008;31:416-69.

12. Wedzicha JA, Decramer M, Seemungal TA. The role of bronchodilator treatment in the prevention of 
exacerbations of COPD. Eur Respir J 2012;40:1545-54.

13. Cazzola M, Calzetta L, Puxeddu E, et al. Pharmacological characterisation of the interaction between glycopyrronium bromide and indacaterol fumarate in human isolated bronchi, small airways and bronchial epithelial cells. Respir Res 2016;17:70.

14. Beeh KM, Beier J. The short, the long and the "ultralong": why duration of bronchodilator action matters in chronic obstructive pulmonary disease. Adv Ther 2010;27:150-9.

15. Barnes PJ. Distribution of receptor targets in the lung. Proc Am Thorac Soc 2004;1:345-51.

16. Kummer W, Krasteva-Christ G. Non-neuronal cholinergic airway epithelium biology. Curr Opin Pharmacol 2014;16:43-9.

17. Wessler I, Kirkpatrick CJ. Acetylcholine beyond neurons: the non-neuronal cholinergic system in humans. Br J Pharmacol 2008;154:1558-71.

18. Donohue JF. Another Choice for Prevention of COPD Exacerbations. N Engl J Med 2016;374:2284-6.

Cite this article as: Cazzola M, Ora J, Puxeddu E. Dual bronchodilation and exacerbations of COPD. J Thorac Dis 2016;8(9):2383-2386. doi: 10.21037/jtd.2016.08.92
19. Bafadhel M, McKenna S, Terry S, et al. Acute exacerbations of chronic obstructive pulmonary disease: identification of biologic clusters and their biomarkers. Am J Respir Crit Care Med 2011;184:662-71.

20. Pascoe S, Locantore N, Dransfield MT, et al. Blood eosinophil counts, exacerbations, and response to the addition of inhaled fluticasone furoate to vilanterol in patients with chronic obstructive pulmonary disease: a secondary analysis of data from two parallel randomised controlled trials. Lancet Respir Med 2015;3:435-42.

21. Segreti A, Stirpe E, Rogliani P, et al. Defining phenotypes in COPD: an aid to personalized healthcare. Mol Diagn Ther 2014;18:381-8.

22. Miravitlles M, Soler-Cataluña JJ, Calle M, et al. Spanish guideline for COPD (GesEPOC). Update 2014. Arch Bronconeumol 2014;50 Suppl 1:1-16.

23. Magnussen H, Disse B, Rodriguez-Roisin R, et al. Withdrawal of inhaled glucocorticoids and exacerbations of COPD. N Engl J Med 2014;371:1285-94. 